\title{
The complex FeK line of the Narrow-Line Seyfert 1 galaxy IRAS 13349+2438
}

\author{
A. L. Longinotti ${ }^{1,2}$, M. Cappi ${ }^{2}$, K. Nandra ${ }^{1}$, M. Dadina ${ }^{2}$, and S. Pellegrini ${ }^{3}$ \\ 1 Astrophysics Group, Imperial College of Science, Technology and Medicine, Prince Consort Rd. SW7 2AZ, London, UK \\ 2 Istituto IASF-CNR, Sezione di Bologna Via Gobetti 101, 40129 Bologna, Italy \\ 3 Universita' degli studi di Bologna, Dipartimento di Astronomia, via Ranzani 1, 40127 Bologna, Italy
}

Received 7 April 2003 / Accepted 24 July 2003

\begin{abstract}
The observation of the Narrow-Line Seyfert 1 galaxy IRAS 13349+2438 performed by XMM-Newton European Photon Imaging Camera between $0.3-10 \mathrm{keV}$, is presented here. The broadband spectrum of the source is dominated at low energies $(E \leq 2 \mathrm{keV})$ by a strong excess of emission and by complex emission/absorption features between $\sim 5.5-8.0 \mathrm{keV}$. The soft X-ray spectrum is consistent with ionized absorption found by the Reflection Grating Spectrometer (Sako et al. 2001a). We focus on the $2-10 \mathrm{keV}$ spectrum which shows clear evidence for a broad, complex FeK $\alpha$ line, previously unseen by ASCA, and for an Fe K-shell edge detected at $\sim 7.3-7.4 \mathrm{keV}$ (rest-frame). The presence of this edge could be explained by either partial covering or ionized/relativistic reflection models, with the latter being preferred and with a resulting power law slope of $\Gamma \sim 2.2$. The line profile is complex, with a broad bump between $\sim 5.5-6.5 \mathrm{keV}$ and a narrow emission line at $\sim 7 \mathrm{keV}$, separated by a sharp drop at $\sim 6.8 \mathrm{keV}$. This profile is compatible with two possible scenarios: i) a broad, ionized and gravitationally redshifted Laor diskline plus a narrow and ionized emission line; ii) a broad, ionized and single Schwarzschild (double-peaked) diskline with a superimposed narrow absorption line.
\end{abstract}

Key words. X-rays: galaxies - galaxies: Seyfert - galaxies: individual: IRAS 13349+2438

\section{Introduction}

It has been recognized that one of the most significant results achieved by the ASCA satellite has been the discovery of broad fluorescent FeK lines in some Seyfert 1 galaxies interpreted as arising from X-ray reflection onto relativistic accretion disks (Tanaka et al. 1995; Fabian et al. 1995; Nandra et al. 1997; Fabian et al. 2000).

The skewed and asymmetric line profiles are consistent with that expected from an accretion disk orbiting a central black hole (Fabian et al. 1989; Stella \& Campana 1991; Matt et al. 1993). The shape and strength of such a component potentially provide a powerful and unique diagnostic of the regions closest to the black hole, but a spectrum of high statistical quality is necessary to obtain any reliable physical conclusion.

Indeed, estimation of the line parameters depends critically on fitting the underlying continuum correctly, as pointed out by, e.g., Pounds \& Reeves (2002) who studied a small sample of Seyfert 1 observed by XMM-Newton. In this regard, they emphasised the importance of analysing EPIC cameras broad-band spectra to identify the primary X-ray continuum because of the relatively large effective area above $7 \mathrm{keV}$ and the opportunity of using the complementary high resolution grating spectrometers, on board both XMM-Newton and Chandra, to resolve and constrain superimposed emission and

Send offprint requests to: A. L. Longinotti, e-mail: all@imperial.ac.uk absorption features (the respective energy bands for these instruments are $0.3-2.5 \mathrm{keV}$ and $0.8-7 \mathrm{keV}$ ).

ASCA results showed that the $\mathrm{FeK} \alpha$ emission line was a common feature in Seyfert 1 galaxies and its peak energy was found to be $\sim 6.4 \mathrm{keV}$ (Nandra et al. 1997). Subsequent Chandra data have indicated that in some cases there is a separate narrow component superimposed on the broad emission (e.g. Yaqoob et al. 2001). In other cases the 6.4 peak may be part of the disk line (Lee et al. 2002; Fabian et al. 2002). In addition, at the time of the writing, there is a considerable uncertainty and discussion as to how common relativistically broadened components are in Seyfert galaxies (e.g. Reeves 2002; Padmanabhan \& Yaqoob 2002). In particular no clear picture has emerged from the new data from XMM-Newton. In some individual cases there is apparently emphatic evidence for relativistic effects in the FeK $\alpha$ line (e.g. Fabian et al. 2002; Turner et al. 2002), while in many others there appears to be no clear evidence for a broad component at all (e.g. Gondoin et al. 2001; Pounds et al. 2002).

IRAS $13349+2438$ is a powerful radio quiet quasar, with a bolometric luminosity $\geq 10^{46} \mathrm{erg} / \mathrm{s}$, at $z=0.10764$. Despite its high luminosity, this source is often classified as a Narrow Line Seyfert 1 (NLS1) for its striking similarities to this class of objects (Brandt et al. 1997). NLS1 galaxies often show extremely complex FeK lines in XMM-Newton observations (Mason et al. 2001; O'Brien et al. 2001; Boller et al. 2002). They are thought to be characterised by high accretion rates 


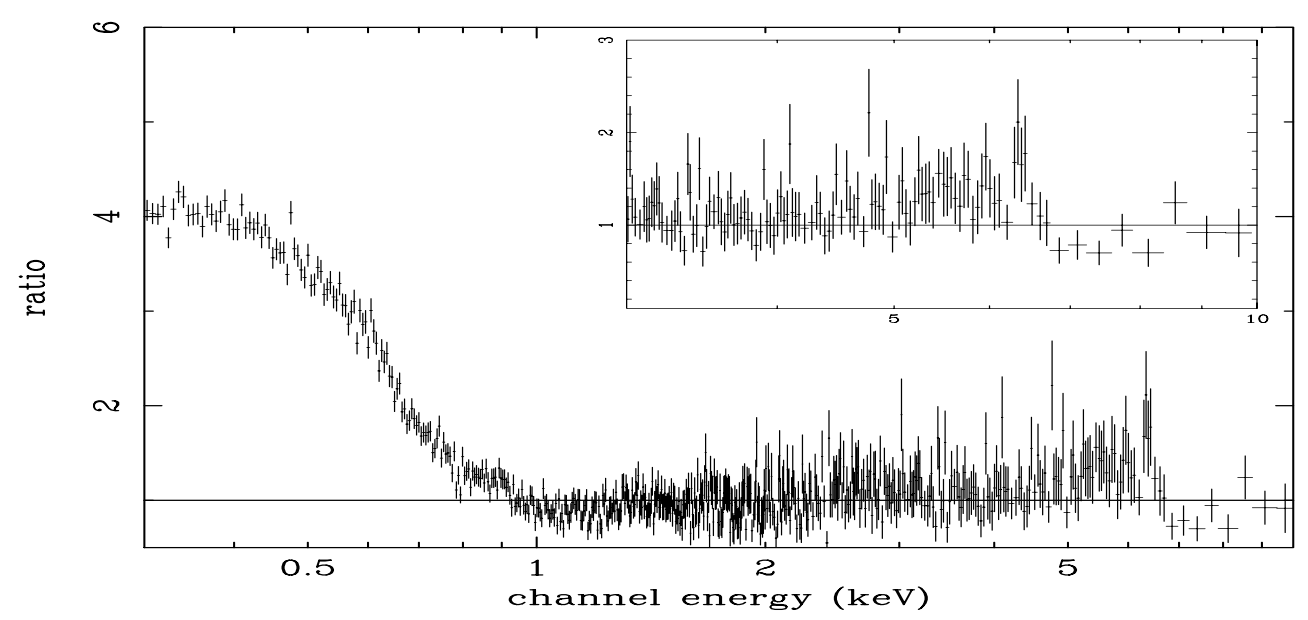

Fig. 1. The XMM-Newton EPIC pn 0.3-10 keV spectrum of IRAS 13349+2438: the figure shows the data to model ratio in the observer's frame. A power-law of slope $2.04 \pm 0.06$ has been fitted to the $2-10 \mathrm{keV}$ data. The extrapolation of this model to lower energies data results in a clear soft X-ray excess. The $3-10 \mathrm{keV}$ residuals in the zoom show a broad hump between $5-6 \mathrm{keV}$, a narrow absorption notch at $\sim 6.2 \mathrm{keV}$ and a narrow feature peaking at $\sim 6.4 \mathrm{keV}$ followed by a deep drop.

(Pounds et al. 1995) which leads to the possibility that the accretion disk is ionized (Ballantyne et al. 2001). IRAS $13349+2438$ 's X-ray spectrum is known to have a steep power-law $(\Gamma \sim 2.2)$ and a soft X-ray warm absorber both in ROSAT and ASCA observations (Brandt et al. 1996, 1997). The warm absorber has been strongly confirmed in the detailed XMM-Newton Reflection Grating Spectrometer (RGS) $0.3-2.5 \mathrm{keV}$ data analysis of Sako and collaborators (Sako et al. 2001a).

Here we report the XMM-Newton EPIC observation of IRAS $13349+2438$, with the intent of exploring the broad-band (0.3-10 keV) spectral properties through the CCD cameras improved sensitivity above $7 \mathrm{keV}$.

\section{Observations and data reduction}

IRAS $13349+2438$ was observed by XMM-Newton on the 19-20 June, 2000 during the Performance Verification phase, for a total duration of $62 \mathrm{ks}$. The data reported here come from the EPIC instrument; the pn camera was used in small window mode with the thin filter, while the MOS1 camera was set in full window with the medium filter. The MOS2 detector was operated in timing mode (FAST UNCOMP) and is not considered here. The data were reduced using the XMM-Newton Science Analysis Software (SAS, version SAS_20001203_1730); the subsequent event selection has been performed taking into account the most recent calibrations. We removed a period of strong contamination by soft protons flare at the end of the light curve, by cutting out all the contaminated events; taking into account also the satellite dead times, the filtered event files result in $44 \mathrm{ks}$ of good exposure for the MOS1 and $31 \mathrm{ks}$ for the pn instrument. We decided to use only pattern 0 events for pn data since the inclusion of higher level patterns reduces the spectral resolution of the instrument, (in these data the pileup percentage can be reduced to $<0.1 \%$ when selecting single events only); for MOS1 data we chose the best calibrated pattern $\leq 12$, for which the pile-up is $\leq \sim 0.01 \%$.
Source events have been collected from a circular region of $40^{\prime \prime}$ of radius centred on the source, while background events have been collected from a similar region in a nearby sourcefree portion of the detectors. For the chosen source radius the encircled energy fraction varies between $88 \%$ and $92 \%$ of the total, from $1.5 \mathrm{keV}$ to $10.5 \mathrm{keV}$. About 93000 source counts were collected from the pn and 33000 from the MOS 1 detector. In the pn the background count rate contributes $\sim 1 \%$ to the source count rate between $0.4-10 \mathrm{keV}$ and less than $10 \%$ between 6-10 keV. We checked for the effect of spatial variations in the EPIC internal background by extracting from multiple areas in the detector, but found no significant difference in the final spectrum. Pulse-height spectra were binned in order to have at least 20 counts/bin which allows the use of $\chi^{2}$ minimization for spectral fitting. Data from the pn camera are used in all the figures but the analysis and the interpretation that follows is also consistent with MOS camera data.

\section{Spectral analysis}

The source flux varied by a factor of $\sim 1.5$ during the XMM-Newton observation. Time resolved hardness ratio analysis did not highlight any significant spectral variation, so we integrated the data over the whole exposure to perform the spectral analysis.

Figure 1 shows the $0.3-10 \mathrm{keV}$ spectrum plotted as the ratio of the observed pn data to a simple power law model fitted to the $2-10 \mathrm{keV}$ data only. The power law slope derived is $\Gamma=2.04 \pm 0.06$. Two striking spectral features clearly stand out from this fit:

- i) an excess of soft X-ray emission below $\sim 1 \mathrm{keV}$;

- ii) a broad feature near the Fe complex at 5-7 keV (the zoom on this feature is also included in Fig. 1).

Parametrization of the $2-10 \mathrm{keV}$ spectrum

The following basic model, called Model 1, aims to describe the spectral shape by parametrizing the data without any physical insight. As a crude parametrization of the $\mathrm{Fe} \mathrm{K} \alpha$ profile, 
we first used two Gaussian emission lines, a narrow blue peak at a rest-frame energy of $E=7.0 \pm 0.1 \mathrm{keV}$, and a broad redshifted "wing" at a rest-frame energy of $E=6.0_{-0.2}^{+0.3} \mathrm{keV}$. An absorption edge at $7.4_{-0.2}^{+0.3} \mathrm{keV}$ rest frame with optical depth of $0.44 \pm 0.22$, also improved the fit in this model by $\Delta \chi^{2}=22$. The fit, with a $\chi^{2}$ of 208 for 216 degrees of freedom and $\Gamma=2.07_{-0.15}^{+0.05}$, gave a photon index of the continuum power-law slightly flatter than $\Gamma=2.2$, as derived from previous ASCA observations (Brandt et al. 1997). The detailed parameters of this model are listed in Table 1 while Fig. 2 shows the unfolded spectrum and the contours at the $68.3 \%$, $90 \%$ and $99 \%$ confidence levels for two interesting parameters of all the three components.

Quick look at the 0.3-10 keV spectrum

In the $0.3-10 \mathrm{kev}$ fitting, Model 1 was fixed based on the 2-10 keV data. The soft X-ray spectrum has been fitted by adding a blackbody component of $k T=85 \pm 1 \mathrm{eV}$ to the power-law. This gives the best description of the soft continuum when compared to other continuum models tried (e.g. a single power-law, a broken power-law and/or a bremsstrahlung model), but still the residuals do require discrete soft X-ray components. We fitted these residuals by adding to the (power law + bbody) model either a traditional warm absorber model or a number of relativistic soft X-ray disklines as recently proposed by several authors (Branduardi-Raymont et al. 2001; Sako et al. 2001b; Mason et al. 2002) for other NLSy1 type objects (very similar to IRAS $13349+2438$ ). The relativistic emission model yields three disklines from O VIII, N VII and a Fe L lines blend. These species are expected from ionized disks, but it is unclear whether their strengths are consistent with theoretical predictions (Ballantyne et al. 2002). The best fit parameters of the warm absorber model, $\xi \sim 12 \mathrm{erg} \mathrm{cm} \mathrm{s}^{-1}$, and $N_{\mathrm{H}} \sim 2.5 \times 10^{21} \mathrm{~cm}^{-2}$ are consistent with the values found by Sako et al. (2001a), based on RGS data (the ionization parameter is defined by $\xi=4 \pi F_{x} / n_{\mathrm{H}}$ ). Both models yielded very large reductions in $\chi^{2}$. We obtained $\Delta \chi^{2}=155$ for the warm absorber model and $\Delta \chi^{2}=255$ for the soft $\mathrm{X}$-ray disklines with respect to the powerlaw+bbody $\chi^{2}=846$ for 562 d.o.f. While both models are physically plausible, these spectral parametrizations are very much model dependent. In reality both components maybe present and higher spectral resolution is required to disengtangle them.

In the following sections we therefore concentrate on the hard X-ray data (above $2 \mathrm{keV}$ ), testing more physically realistic models than Model 1, which can in principle account simultaneously for the narrow and broad residuals between 5-7 keV, and for the Fe-K absorption edge.

\subsection{Origin of the Iron-K edge: transmission models}

The simple parametrization described before, includes a strong $\mathrm{Fe}-\mathrm{K}$ edge at $\sim 7.4 \mathrm{keV}$. The physical origin of this edge can be important in the interpretation of the remainder of the spectrum (e.g. Pounds \& Reeves 2002; Fabian et al. 2002). An origin in line-of-sight material was first considered. This can account for the edge, but could also cause apparent curvature in the continuum mimicking a broad disk line at lower energies.
The edge optical depth and energy in Model 1 are well constrained; the energy of $7.4 \mathrm{keV}$ implies the Iron plasma is mildly ionized (Fe X-XII). The equivalent $\mathrm{H}$-column density of the absorbing medium estimated from the Fe K-shell cross section and from the observed edge's $\tau$, results in $N_{\mathrm{H}} \sim$ $2.8 \times 10^{23} \mathrm{~cm}^{-2}$. As we would then expect an Iron L-edge at $\sim 0.95-1.03 \mathrm{keV}$ rest frame, we calculated the photoabsorption cross sections of K-shell and L-shell for that particular ionization stage, using a procedure by Verner et al. (1993). From the $N_{\mathrm{H}}$ value and the L-shell cross section, the expected absorption L-edge optical depth is therefore estimated as $\tau=8.6$. No edge with such optical depth is detected. A total covering absorber therefore cannot account for the FeK-edge, so the feasibility of a partial covering model was tested. In general the column can be ionized so we combined the XSPEC ABSORI model (Zdziarski et al. 1990; Done et al. 1992) with two power laws, one unabsorbed and one subject to ionised absorption (reproduced by the ABSORI model). If we are to mimic an edge with $\tau=0.4$ in the total spectrum, this sets a minimum value for the covering fraction. Even if the optical depth at the Fe K-edge in the absorbed component, $\tau_{\text {abs }}$, is infinite, the edge in the combined spectrum cannot be reproduced unless the covering fraction is greater than $\mathrm{e}^{-\tau_{\text {abs }}}$. We therefore set a lower limit to the covering fraction of $C_{\mathrm{f}}>0.66$. Such a model in principle mimics the spectrum broad hump and the absorption edge, reproducing the effect of a "patchy" absorber where the nuclear emission leaks through a material of column density $N_{\mathrm{H}}$. The same Gaussian narrow line of Model 1 at $\sim 7 \mathrm{keV}$ is included in this fit to account for the narrow peak residuals.

The fit with $\mathrm{Fe}$ abundance fixed to the solar value ${ }^{1}$ gave $\chi^{2}=232 / 218$ degrees of freedom, with photon index of $2.67_{-0.11}^{+0.08}$, column density of $N_{\mathrm{H}}=7.2 \times 10^{22} \mathrm{~cm}^{-2}$; by leaving the $\mathrm{Fe}$ abundance free to vary during the fit, it becomes greater than the solar value by a factor of 12 and the $\mathrm{H}$ column density decreases to $3.47 \times 10^{22} \mathrm{~cm}^{-2}$, with $\Gamma=2.66_{-0.09}^{+0.10}$. Figure 3 shows this over-abundant fit, which, although still worse than Model 1, gave a statistical improvement $\left(\chi^{2}=\right.$ $226 / 217$ degrees of freedom) compared with the one with solar Iron abundance. Such Fe abundance is however hard to explain. Mildly ionized gas partially covering the X-ray source can therefore account for the Iron edge only if it is highly overabundant in Iron, and in any event does not account well for the broad residuals in the 5-7 keV band, where a broad emission feature is required by the data. An alternative origin for both line and edge is in reflection. We now consider such models.

\subsection{Origin of the Iron K-edge: Reflection models}

Another possible origin for the edge feature at $7.4 \mathrm{keV}$ is via reflection in optically thick material (George \& Fabian 1991; Matt et al. 1991). Indeed, any emission line produced by optically thick material should be accompanied by a reflection continuum, as is commonly observed (e.g.

\footnotetext{
1 The abundances have been set to the values given by Anders \& Grevesse (1989); in particular, Fe abundance is normalized to the solar value of $4.68 \times 10^{-5}$ with respect to hydrogen.
} 
Table 1. Double component models fitted on the 2-10 keV spectrum, respectively illustrated in Figs. 2, 5 and 4; all the energies are reported in the source rest-frame.

\begin{tabular}{|c|c|c|c|c|c|c|c|c|c|c|c|c|}
\hline Model & $\Gamma$ & $R$ & $\begin{array}{c}E_{\mathrm{b}} \\
(\mathrm{keV})\end{array}$ & $\begin{array}{l}E W_{\mathrm{b}} \\
(\mathrm{eV})\end{array}$ & $\begin{array}{c}\sigma_{\mathrm{b}} \\
(\mathrm{keV})\end{array}$ & $\begin{array}{c}E_{\mathrm{n}} \\
(\mathrm{keV})\end{array}$ & $\begin{array}{l}E W_{\mathrm{n}} \\
(\mathrm{eV})\end{array}$ & $\begin{array}{c}E_{\mathrm{abs}} \\
(\mathrm{keV})\end{array}$ & $E W_{\text {abs }}$ & $E_{\text {edge }}$ & $\tau$ & $\chi^{2} /$ d.o.f. \\
\hline & (1) & (2) & (3) & (4) & (5) & (6) & (7) & (8) & (9) & (10) & (11) & (12) \\
\hline $\begin{array}{l}\text { (1) Two Gaussians }+ \\
\text { iron edge }\end{array}$ & $2.07_{-0.15}^{+0.05}$ & - & $6.0_{-0.2}^{+0.3}$ & $409_{-370}^{+105}$ & $0.48_{-0.12}^{+0.32}$ & $7.0 \pm 0.1$ & $123_{-110}^{+62}$ & - & - & $7.4_{-0.2}^{+0.3}$ & $0.44 \pm 0.22$ & 208/216 \\
\hline $\begin{array}{l}\text { (2) Sch. diskline + } \\
\text { narrow abs.line }+ \\
\text { iron edge }\end{array}$ & $2.24_{-0.08}^{+0.010}$ & $2.3_{-0.8}^{+0.8}$ & $6.84_{-0.26}^{+0.29}$ & $535_{-298}^{+367}$ & - & - & - & $6.86_{-0.14}^{+0.07}$ & $107_{-82}^{+79}$ & $7.27_{-0.44}^{+0.19}$ & $4.3_{-1.9}$ & $211 / 212$ \\
\hline $\begin{array}{l}\text { (3) Kerr diskline + } \\
\text { narrow em. line + } \\
\text { iron edge }\end{array}$ & $2.24_{-0.11}^{+0.11}$ & $2.09_{-0.95}^{+0.89}$ & $6.67_{-0.67}^{+0.72}$ & $179_{-189}^{+190}$ & - & $7.02_{-0.06}^{+0.25}$ & $110_{-110}^{+130}$ & - & - & $7.26_{-0.64}^{+0.15}$ & $5.5_{-2.0}$ & $214 / 212$ \\
\hline
\end{tabular}

(1) Power law photon index.

(2) Reflection fraction i.e. the ratio between the normalization of the reflected and direct component, $R=A_{\text {refl }} / A_{\mathrm{pl}}$.

(3) Source rest frame energy of the broad line.

(4) Broad line equivalent width, measured with respect to the total continuum flux (i.e. from the sum of power law and reflection); the large errors are due to the uncertainties in estimating $\mathrm{X}$-ray line fluxes in the XSPEC package.

(5) Width of the broad line in the parametrization model 1; notice that the edge width in the SMEDGE model has been fixed to this value, regarded as an approximate measure of the features smearing.

(6) Source rest frame energy of the narrow Gaussian emission line and (7) its $E W$ (with respect to the total continuum).

(8) Source rest frame energy of the resonant absorption line.

(9) $E W$ of the res. abs. line, measured from the total continuum.

(10) Source rest frame energy of the FeK absorption edge, fitted by an EDGE component in model 1 and by a SMEDGE component in 2 and 3.

(11) Optical depth of the FeK edge; the errors in model 2 and 3 give a lower limit only.

(12) $\chi^{2}$ and degrees of freedom of the fit; parameters fixed in model 2 and 3: width of the edge; abundances, disk temperature $\left(10^{4} \mathrm{~K}\right)$, cos $i$. 

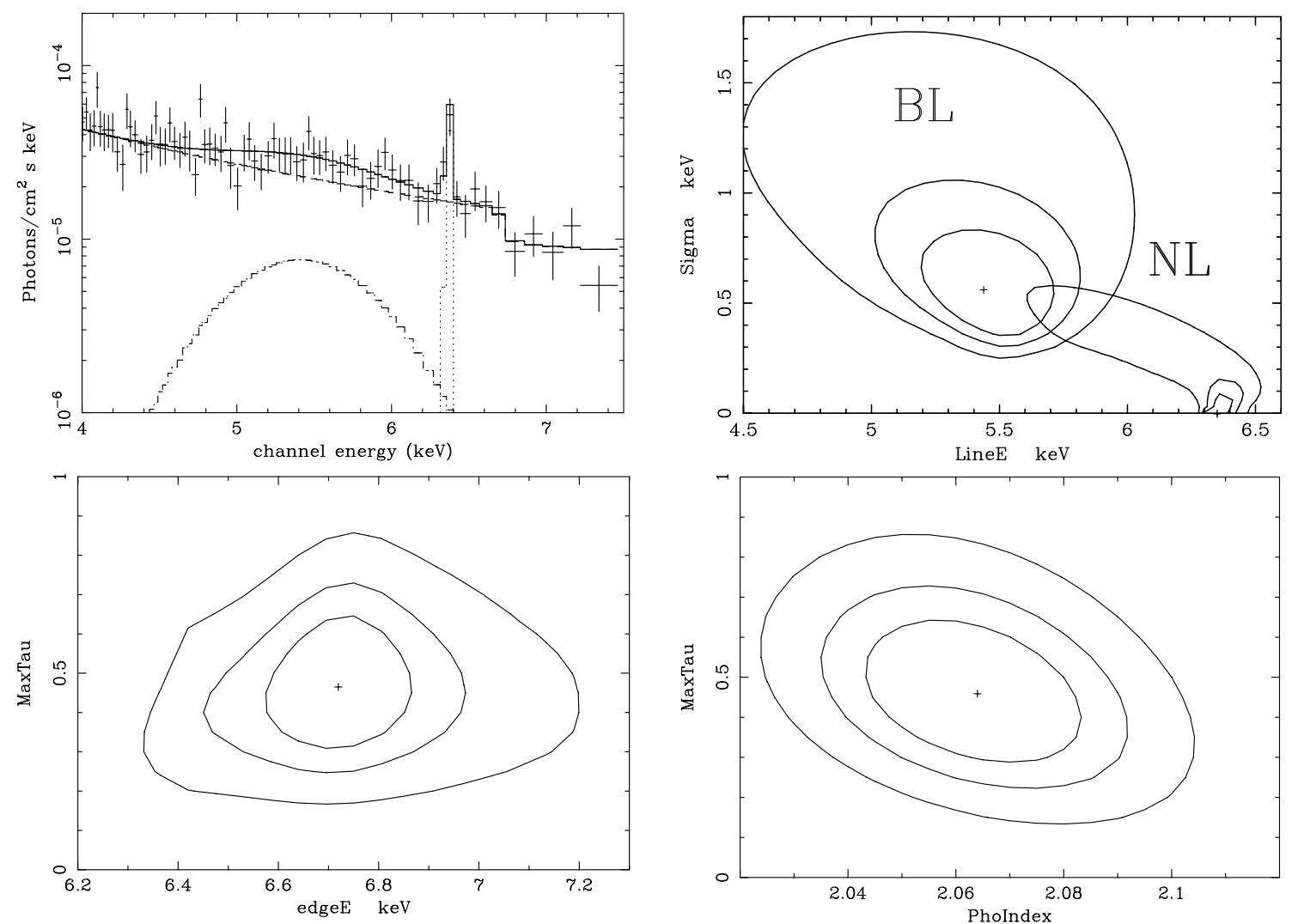

Fig. 2. Model 1: the $2-10 \mathrm{keV}$ data have been parametrized by a simple model consisting in a power-law of $\Gamma=2.07_{-0.15}^{+0.05}$, two Gaussian emission lines and an absorption edge; left above: observer's frame picture of the unfolded spectrum shows the data fitted by the underlying model; clockwise: contours at the 68\%, 90\% and 99\% confidence level for energy and width of both the broad and the narrow lines, for the power-law photon index and the edge optical depth, and for the edge energy and optical depth. All the energies in the picture are in the observer's frame, for a complete list of parameters, see Table 1.

Nandra \& Pounds 1994). Having established the presence of reprocessing by mildly ionized gas because of the energies of the features in Model 1, three different XSPEC reflection models from ionized material have been tried for fitting the continuum. In all of them, the reflection fraction $R=A_{\text {refl }} / A_{\mathrm{pl}}$ expresses the amount of reflection with respect to the incident X-ray irradiating flux and it is usually assumed to be descriptive of the source geometry ( $R=\Omega / 2 \pi, R \sim 1$ if the reprocessing material covers $2 \pi$ of the source). We first used the model PEXRIV (Magdziarz \& Zdziarski 1995). It turned out that the confidence region for the reflection fraction is strongly biased towards very high values of $R(\sim 4)$ with a photon index of $\sim 2.2$ assuming a surface temperature of $3 \times 10^{4} \mathrm{~K}$, in an optically thick disk, with an average inclination angle of $\sim 25^{\circ}$.

When $R$ is $\geq 2$, anisotropy and/or time delay effects are implied, but presumably in this case the present data have a large value of $R$ because the reflection is forced to fit the deep drop of the spectrum at energy greater then $\sim 7 \mathrm{keV}$. Relativistic effects are very likely to be involved if the edge originates in an accretion disk, as suggested by the broad hump in Fig. 1 assuming this is identified with a disk line. These effects not only broaden but shift the apparent edge threshold energy, which may result in a poor estimate for the ionization state and optical depth. To overcome these problems a smoothed edge component (SMEDGE) was added to the model after setting to zero the value of the $\mathrm{Fe}$ abundance in the PEXRIV; in this way only the SMEDGE accounts for the drop, mimicking the relativistic smearing. Fits with the PEXRIV/SMEDGE model alone were, perhaps unsurprisingly, unable to account for the broad disk-line residuals seen at 5-7 keV. We therefore added a diskline separately. This alternative model leads to an estimation of the reflection fraction of $R \sim 2$ and $\Gamma \sim 2.24$, with an inclination for the disk of $\sim 30^{\circ}$, values which are certainly more common to Seyfert 1. The energy of the edge is not affected very much by the type of line, being $7.27_{-0.44}^{+0.19} \mathrm{keV}$ with a Schwarzschild diskline and $7.26_{-0.64}^{+0.15} \mathrm{keV}$ with a Laor diskline, see Table 1 for a complete list of parameters and $\chi^{2}$. The PEXRIV/SMEDGE models have an excellent fit, depending on the line model (see below). Surprisingly, more physically realistic reflection models for the edge did not match the data particularly well. We tried both the REFSCH and XION models, which are computations of a power law reflected on an ionized accretion disk, convolved with a relativistic disk line, (see Magdziarz \& Zdziarski 1995; Nayakshin et al. 2001), but the shape of the spectrum in both cases does not model the edge residuals very well and there is no statistical improvement $\left(\chi^{2}=239 / 218, \chi^{2}=236 / 216\right.$ respectively $)$. 
Table 2. Best fit relativistic disklines parameters in model 2 and 3; the ones with no errors have been fixed to the bestfit values in order to avoid degeneration.

\begin{tabular}{cccccc}
\hline \hline Model & $E$ & $\beta$ & $R_{\text {in }}$ & $R_{\text {out }}$ & $i$ \\
& $\left(R_{\mathrm{g}}\right)$ & $\left(R_{\mathrm{g}}\right)$ & $(\mathrm{deg})$ \\
\hline Schwarzschild Diskline & $6.84_{-0.26}^{+0.29}$ & 2 & 6 & 28 & 28 \\
Laor Diskline & $6.67_{-0.67}^{+0.72}$ & 1.9 & 1.36 & 25 & 17 \\
\hline
\end{tabular}

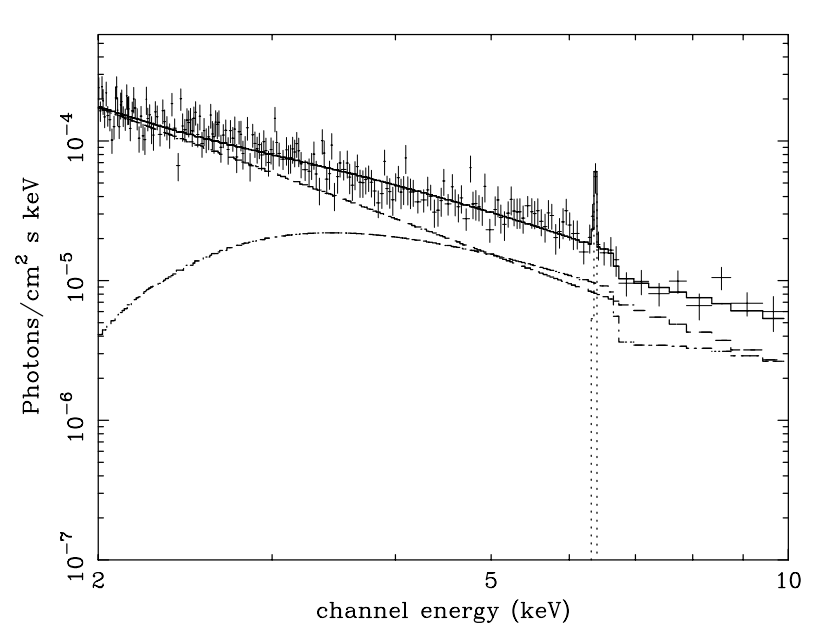

Fig. 3. Observer's frame partial covering model with over abundance of Iron: the broad residuals at $\sim 5-6 \mathrm{keV}$ are still clearly visible and there is no evidence of absorption in the data below $3 \mathrm{keV}$, as required by the column density of a few $\times 10^{22} \mathrm{~cm}^{-2}$.

\subsection{Origin of the broad $5-7 \mathrm{keV}$ feature}

Neither complex absorption nor reflection appears to account well for the broad feature between 5 and $7 \mathrm{keV}$ (Fig. 1). It therefore seems most likely that this originated as a relativistic disk line, associated with the reflection spectrum discussed above. We tested models in both the Schwarzschild and Kerr metrics (Fabian et al. 1989; Laor et al. 1991). The results are shown in Table 1. Our investigations of various line models resulted in two possibilities, model 2 and model 3 in Table 1, respectively with disk line in Schwarschild metric or Kerr metric. Disk line models provide a good fit to the red wing of the data; such models consist in several parameters and so they are usually affected by considerable degeneracy. The model complexity does not allow to constrain all the parameters, but if the inner radius is left free to vary, it clearly tends to its minimum value $\left(6 R_{\mathrm{g}}\right)$ in Schwarzschild case. With this low value, the Fe line emission is strongly concentrated in the central regions, which suggests that the disk extends closer to the black hole where the relativistic effects are most important. The Kerr metric allows to extend the emissivity region to the last stable orbit $\left(1.23 R_{\mathrm{g}}\right)$ and the inner radius reaches such low values that it has been fixed at its minimum in order to avoid local minima for the $\chi^{2}$ process. The same procedure has been adopted for the emissivity law index (emissivity scales as $R^{-\beta}$ ) which tends to $\beta \sim 2$ in both line models and it is kept fixed. For the sake of simplicity, a separate table (Table 2 ) lists the relativistic line parameters of the best fits 2 and 3; the outer radii and the disk

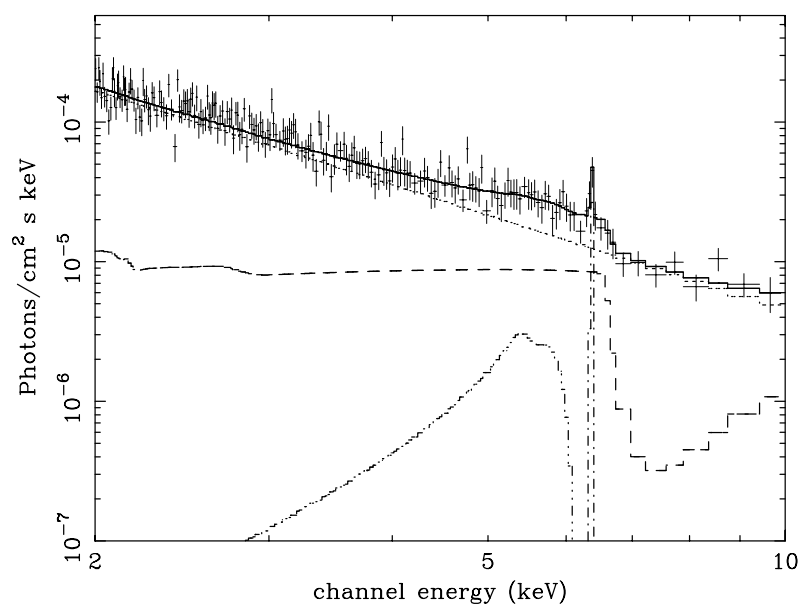

Fig. 4. Observer's frame picture of the unfolded spectrum for model 3 of Table 1 with the $\mathrm{FeK} \alpha$ fitted by a Kerr diskline plus a narrow Gaussian emission line (the rest frame energies are respectively $6.67_{-0.67}^{+0.72} \mathrm{keV}$ and $7.02_{-0.06}^{+0.25} \mathrm{keV}$ ).

inclination angles span a wide range of values so, again, we decided to fix them at the values most commonly given by the fitting.

However, the line profile cannot be well fitted with a single component, even considering relativistic effects for every spectral feature, as done so far; such models are inadequate to fit the "sharp" notch at $E \sim 6.2 \mathrm{keV}$ (observer's frame, Fig. 1); we were thus forced to use double-component solutions, listed in Table 1 and illustrated in Figs. 4 and 5. A narrow Gaussian emission line was added to the Laor disk line (model 3), obtaining a $\chi^{2}$ of 214/212 degrees of freedom (Fig. 4). The rest frame energies of both the lines are very ionized, being $6.67_{-0.67}^{+0.72} \mathrm{keV}$ for the broad one and $7.02_{-0.06}^{+0.25} \mathrm{keV}$ for the narrow one. Then, the next double component model consists in a Schwarzschild disk line plus a narrow Gaussian absorption line with $\sigma$ fixed at $0 \mathrm{keV}$ and negative intensity, in order to account for the narrow absorption notch, visible at $6.2 \mathrm{keV}$ (observer's frame) in Fig. 1 (zoom). The absorption line energy is $6.86_{-0.14}^{+0.07} \mathrm{keV}$, which is just slightly different from the peak energy of the broad one, $E=6.84_{-0.26}^{+0.29} \mathrm{keV}$. The confidence contours in Fig. 6 show that the absorption line is well constrained and it improves the fit by $\Delta \chi^{2}=10$ for two free parameters, i.e. $\geq 99 \%$ of significance. This model provides the best fit of all the reflection ones, giving a $\chi^{2}=211 / 212$ and is the one shown in Fig. 5.

While we disfavour above the possibility that the Fe K-edge arises from a fully or partially covering absorber, there is nonetheless clear evidence in the RGS spectrum for ionized 


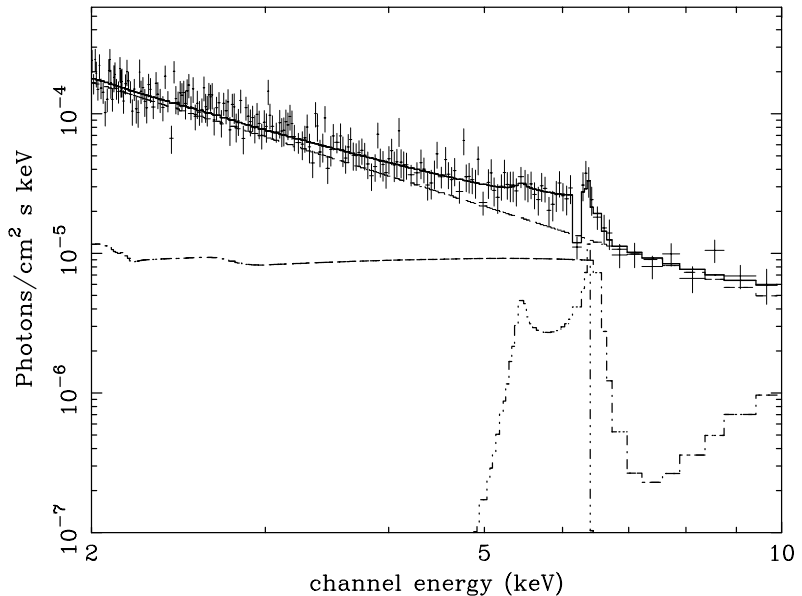

Fig. 5. Observer's frame picture of the unfolded spectrum for model 2 in Table 1. The rest frame energies are $6.84_{-0.15}^{+0.17} \mathrm{keV}$ for the Schwarschild diskline and $6.86_{-0.10}^{+0.08} \mathrm{keV}$ for the resonant absorption line superimposed on the previous one.

gas in line-of-sight to IRAS 13349+2438 (Sako et al. 2001a). Such a component may affect our fits if it has a sufficiently high column density. The highest column component identified by Sako et al. has $N_{\mathrm{H}} \sim(1-4) \times 10^{22} \mathrm{~cm}^{-2}$ with an ionization parameter in the range $2.0 \leq \log \xi \leq 2.5$. This is indeed high enough to have a small effect on the spectrum around $2 \mathrm{keV}$ and could change the parametrization of the line. We have therefore added a completely covering ABSORI component to the above fits. This provides a significant improvement of the fit, yielding a $\chi^{2}=200 / 211$ d.o.f. The continuum photon index is much more steeper than previous models, being $\Gamma=2.67_{-0.07}^{+0.20}$, and the reflection fraction is $R=1.13_{-0.27}^{+0.28}$. The power law is absorbed by a column of ionized gas $N_{\mathrm{w}} \sim(2-5) \times 10^{22} \mathrm{~cm}^{-2}$ and the ionization parameter of the absorbing medium is distributed in a range $1 \leq \log \xi \leq 2$, consistent with the value inferred by RGS data by Sako et al. (2001a). The FeK edge optical depth is considerably lower than found before, $\left(\tau=2.07_{-1.07}^{+2.13}\right)$, probably due to the higher photon index, while the energy is still $7.30 \pm 0.28 \mathrm{keV}$. There is very little affect on the line parameters, however, and we therefore conclude that the ionized absorption inferred by Sako et al. (2001a) in this objects will not change our interpretation of the Fe-complex significantly.

\section{Discussion}

\subsection{On the hard $X$-ray continuum and strength of the Fe-K features}

The properties of IRAS $13349+2438$ EPIC spectrum are discussed, mainly concentrating on the Iron line region. The X-ray spectrum turns out to be at least unusual, if not ambiguous. Throughout the spectral analysis, the Fe K-edge is used as a key to understand which is the underlying physical model. Its energy at $\sim 7.3-7.4 \mathrm{keV}$ indicates a medium degree of ionization (Fe VIII-XII); from the spectral fitting a transmission edge is ruled out, as the transmission models in Sect. 3.1 clearly do not provide a good fit for the $5-7 \mathrm{keV}$ residuals and also they require a very high Iron over abundance (see Fig. 3). Moreover,

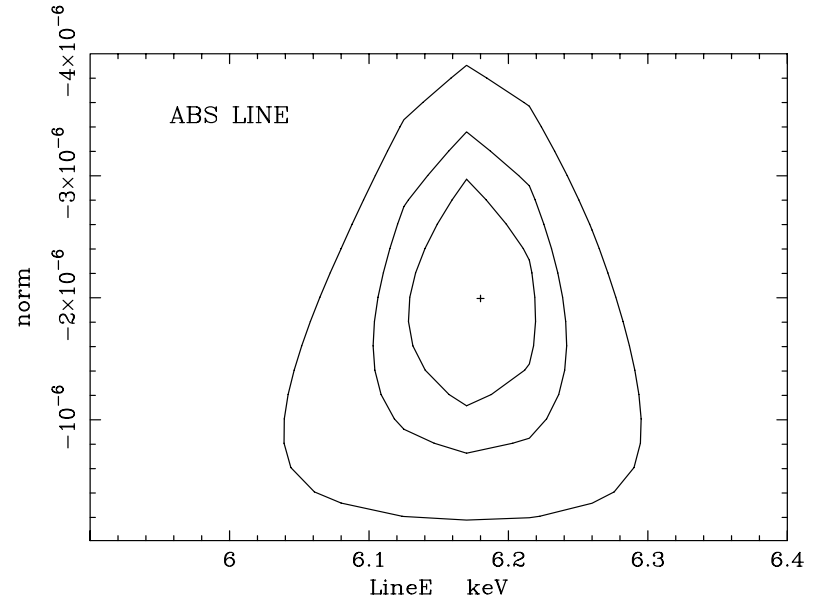

Fig. 6. Observer's frame confidence contours at $68 \%, 90 \%$ and $99 \%$ confidence level for the energy and the intensity of the narrow absorption line in Model 2: the absorption line is plotted with negative intensity.

we would expect an almost neutral Fe emission line associated to the photoabsorption edge, produced by transmission through the medium along our line of sight: the Iron absorbs the primary radiation coming from the central source and re-emits X-ray photons by fluorescence correspondently to its ionization state. But this is not consistent with our case since we do not detect any neutral or near-neutral emission line.

The next hypothesis of a reflection Iron edge has brought us to use reflection models for the continuum, which can also fit the 5-7 keV "bump" in the data with the associated $\mathrm{FeK} \alpha$ emission. Recent publications have stated the opportunity of using self-consistent ionized disk models which can fit simultaneously the reflection continuum and the Iron features (Nayakshin \& Kallman 2001; Ballantyne et al. 2001).

Nevertheless we found that the more phenomenological PEXRIV/SMEDGE model gave a better fit, due to the complexity of the Fe line/edge region and the consequent necessity of an ad hoc, (and hence flexible) model ${ }^{2}$. The photon index derived after adding the reflection component, $(\Gamma \sim 2.2)$, is consistent with the one found by ASCA (Brandt et al. 1997) and with the mean value of $\Gamma$ estimated by Leighly (1999) for 23 NLS1. As expected, once the spectrum is fitted with the reflection continuum, the power law slope results steeper than it is in Model 1 by $\Delta \Gamma \sim 0.18$ (Nandra \& Pounds 1994). The reflection fraction includes the uncertain information concerning the geometry of source and reflector. We find $R=1-2$, depending on the continuum parametrization. This strong reflection is primarily driven by the deep edge in the data. It is consistent with the high equivalent width of the line $(E W \sim 530 \mathrm{eV})$, with the latter also being enhanced by the high ionization state implied by the line energy. High reflection fractions have already been found in Seyfert 1 galaxies and cannot be excluded a priori (Matt et al. 2003). Nonetheless it is troubling that we

\footnotetext{
${ }^{2}$ Using diskline models as we did, allows a greater control on the different components and on the line profile shape, which is affected by changing all the parameters in Table 2 .
} 
have been unable to find a self-consistent physical model which simultaneously account for both the line and the deep edge.

In this regard, an interesting possibility has been suggested by Fabian et al. (2002). In their model the spectrum is reflection-dominated as a result of multiple reflections from different layers of cold material, likely to be produced in highaccretion rate systems as NLS1 as a consequence of accretion instabilities. They find a good fit to another NLS1 with a deep edge, 1H0707-49 (Boller et al. 2002). We attempted to fit the broadband X-ray spectrum of IRAS $13349+2438$ with such model using a combination of three reflectors plus a blackbody component of $k T \sim 76 \mathrm{eV}$ to account for the thermal soft $\mathrm{X}$-ray emission from the disk. Each reflector is modeled using a constant-density ionized disk model (Ross \& Fabian 1993; Ballantyne et al. 2001) on which relativistic blurring is applied appropriate to a maximally spinning black hole. A narrow Gaussian emission line at $\sim 7 \mathrm{keV}$ rest frame is also included in the fit. This model is not only able to account for the line and edge features in IRAS $13349+2438$, but provides a quite reasonable fit to the entire broadband spectrum $(0.3-10 \mathrm{keV}$; $\chi^{2}=675 / 545$ d.o.f); The three reflectors have all different ionization parameters indicating the variously ionized layers in the disk: $\log \xi=1.977$, with $R_{\text {in }}=2.08 R_{\mathrm{g}}, \log \xi=3.957$ with $R_{\text {in }}=13 R_{\mathrm{g}}, \log \xi=1.494$ with $R_{\text {in }}=23 R_{\mathrm{g}}$ going towards the outer disk. This fascinating possibility merits further exploration with future data.

\subsection{On the FeK line structure}

The FeK $\alpha$ diskline is surely the most interesting feature in the $2-10 \mathrm{keV}$ spectrum of IRAS $13349+2438$. It is clearly well modelled by the superposition of two components, for which two scenarios have been found: i) the sum of two emission lines or ii) a single, double-peaked diskline plus a narrow absorption line. Disk models give an excellent fit to the broad red wing and strongly indicate emission coming from the accretion disk inner regions. From these data we cannot distinguish between rotating and nonrotating black hole models, although a Kerr geometry seems more likely as it allows the emitting region extending very close to the black hole. In every model, the peak rest energy $(6.7-6.9 \mathrm{keV})$ implies the presence of Iron atoms at a very high ionization level (Fe XXIII-XXVI) in the accretion disk. Such high states can account for the high $E W$ of the line, as there is a considerable enhancement of the fluorescence mechanism corresponding to Fe XX-Fe XXVI (Zycki \& Czerny 1994) and also a ionized reflection is likely to produce such a strong line for particular source geometries (Matt et al. 1993).

In case i) the broad hump at $5-6 \mathrm{keV}$ in Fig. 1, is fitted by an emission diskline in Kerr metric, whereas the narrow peak at $\sim 6.3 \mathrm{keV}$ in the same figure, is accounted by a second emission line, which is very ionized. The current interpretation for a narrow Fe $\mathrm{K}$ emission line as being due to fluorescence from material distant from the inner disk region, seems the most appropriate. One possibility of producing the emitted Iron line is reflection onto optically thin material, far from the central region, for which the so-called warm mirror might be a candidate
(Krolik \& Kallman 1987). Improved measurements of the line width and flux variability will better constrain the origin of the narrow $\mathrm{Fe} \mathrm{K} \alpha$.

In the ii) hypothesis, the red hump plus a narrow emission peak are interpreted as a single Schwarzschild diskline and the narrow absorption component at $6.8 \mathrm{keV}$ that is produced by resonant absorption; this is a scattering process caused by Hydrogen- and Helium-like Fe ions which resonantly absorb the fluorescent Iron line and continuum photons. The resonant transitions are expected to occur at the energies of $6.7 \mathrm{keV}$ and $6.9 \mathrm{keV}$. The total Iron line profile is then influenced by the absorption feature.

Revealing and studying with greater details such resonant absorption lines is a key point in determining not only the $\mathrm{FeK} \alpha$ line profile, but also the accretion disk environment. In fact, the first case was a very redshifted absorption feature found in the Seyfert 1 NGC 3516 by Nandra et al. (1999), which represents a tentative and rare direct evidence for material actually accreting onto the black hole in active galactic nuclei. Theoretical calculations of the resonant absorption line $E W$ are available in Matt et al. (1997). It is also of note that resonant absorption features in the Fe K region are now becoming detectable even with low resolution in XMM-Newton EPIC spectra (Pounds et al. 2003); when redshifted, they might be observable in brighter objects with higher precision with long exposure of Chandra gratings. The IRAS 13349+2438 resonant line, although not redshifted, shows the existence of a plasma of $\mathrm{Fe}$ ions placed somewhere above the corona, capable of imprinting significant absorption features on AGN spectra. This gas is not to be confused with the soft X-ray warm absorbers found by Sako et al. which are much less ionized; it might be a signature of a thin skin or wind sourrounding the accretion disk (Ruszkowski \& Fabian 1999). Variability studies of the line profile could be a powerful tool for constraining the size and position of this component and accurate measurements of the absorption line width could provide valuable information on the velocity gradient within the gas. For meaningful further progress, future observations need both high resolution and good signal-to-noise ratio. Observations with Astro-E 2 and later Constellation- $\mathrm{X}$ are therefore best suited to discriminate between the possible scenarios for the origin of the Fe line complex in this galaxy.

Acknowledgements. We thank the XMM-Newton Calibration Team for contributing to the operations of the satellite and for continuous maintenance of the software and the anonymous referee who contributed to improve this paper. Financial support from the POE network at the Imperial College, London, from Italian Space Agency by contracts N.ASI/I/R/107/00 and I/R/042/02, and from Angelo Della Riccia Foundation is acknowledged. The authors wish to thank Prof. Andy Fabian for providing the relativistic blurring codes. A.L. Longinotti is pleased to thank Cristian Vignali for his constructive comments and suggestions.

\section{References}

Ballantyne, D. R., Iwasawa, K., \& Fabian, A. C. 2001, MNRAS, 323, 506

Ballantyne, D. R., Ross, R. R., \& Fabian, A. C. 2002, MNRAS, 336, 867 
Boller, T., Fabian, A. C., Sunyaev, R., et al. 2002, MNRAS, 329, L1

Brandt, W. N., Fabian, A. C., \& Pounds, K. A. 1996, MNRAS, 278, 326

Brandt, W. N., Mathur, S., \& Elvis, M. 1997a, MNRAS, 285, L25

Brandt, W. N., Mathur, S., Reynolds, C. S., \& Elvis, M. 1997b, MNRAS, 292, 407

Branduardi-Raymont, G., Sako, M., Kahn, S. M., et al. 2001, A\&A, 365, L140

Done, C., Mulchaey, J. S., Mushotzky, R. F., \& Arnaud, K. A. 1992, ApJ, 395, 275

Fabian, A. C., Rees, M. J., Stella, L., \& White, N. E. 1989, MNRAS, 238,729

Fabian, A. C., Nandra, K., Reynolds, C. S., et al. 1995, MNRAS, 277, L11

Fabian, A. C., Iwasawa, K., Reynolds, C. S., \& Young, A. J. 2000, PASP, 112, 1145

Fabian, A. C., Vaughan, S., Nandra, K., et al. 2002, MNRAS, 335, L1

Fabian, A. C., Ballantyne, D. R., Merloni, A., et al. 2002, MNRAS, 331, L35

George, I. M., \& Fabian, A. C. 1991, MNRAS, 249, 352

Gondoin, P., Lumb, D., Siddiqui, H., Guainazzi, M., \& Schartel, N. 2001, A\&A, 373, 805

Krolik, J. H., \& Kallman, T. R. 1987, ApJ, 320, L5

Laor, A. 1991, ApJ, 376, 90

Lee, J. C., Ogle, P. M., Canizares, C. R., et al. 2001, ApJ, 554, L13

Leighly, K. M. 1999, ApJS, 125, 317

Magdziarz, P., \& Zdziarski, A. A. 1995, MNRAS, 273, 837

Makishima, K. 1986, LNP Vol. 266: The Physics of Accretion onto Compact Objects, 249

Mason, K. O., Branduardi-Raymont, G., Ogle, P. M., et al. 2002 [astro-ph/0209145]

Matt, G., Fabian, A. C., \& Ross, R. R. 1993, MNRAS, 262, 179

Matt, G., Fabian, A. C., \& Reynolds, C. S. 1997, MNRAS, 289, 175

Matt, G., Guainazzi, M., \& Maiolino, R. 2003, Changing look: from Compton-thick to Compton-thin, or the re-birth of fossil AGN [astro-ph/0302328]

Nandra, K., \& Pounds, K. A. 1994, MNRAS, 268, 405

Nandra, K., George, I. M., Mushotzky, R. F., Turner, T. J., \& Yaqoob, T. 1997, APJ, 477, 602

Nandra, K., George, I. M., Mushotzky, R. F., Turner, T. J., \& Yaqoob, T. 1999, ApJ, 523, L17
Nayakshin, S., \& Kallman, T. R. 2001, ApJ, 546, 406

O'Brien, P. T., Page, K., Reeves, J. N., et al. 2001, MNRAS, 327, L37

Padmanabhan, U., \& Yaqoob, T. 2002, New Chandra Results on Seyfert I galaxies: Fe-K lines [astro-ph/0211386]

Pounds, K. A., Done, C., \& Osborne, J. P. 1995, MNRAS, 277, L5

Pounds, K., \& Reeves, J. 2002, What can we learn from EPIC X-ray spectra of Seyfert 1 galaxies? [astro-ph/0201436]

Pounds, K. A., Reeves, J. N., Page, K. L., et al. 2002, A simultaneous XMM-Newton and BeppoSAX observation of the archetypal Broad Line Seyfert 1 galaxy NGC 5548 [astro-ph/0210288]

Pounds, K. A., Reeves, J. N., King, A. R., et al. 2003, A high velocity ionised outflow and soft X-ray photosphere in the narrow emission line quasar PG1211+143 [astro-ph/0303603]

Reeves, J. 2002, Active Galactic Nuclei: From Central Engine to Host Galaxy Abstract Book, meeting held in Meudon, France, July 23-27, 2002, ed. S. Collin, F. Combes, \& I. Shlosman, to be published in ASP Conf. Ser., p. 7

Ross, R. R., \& Fabian, A. C. 1993, MNRAS, 261, 74

Ruszkowski, M., \& Fabian, A. C. 2000, MNRAS, 315, 223

Sako, M., Kahn, S. M., Behar, E., et al. 2001a, A\&A, 365, L168

Sako, M., et al. 2001b, ApJ, to be published [astro-ph/0112436]

Stella, L., \& Campana, S. 1991, Iron Line Diagnostics in X-ray Sources, Proceedings of a Workshop Held in Varenna, Como, Italy, 9-12 October 1990, ed. A. Treves, G. C. Perola, \& L. Stella, (Berlin, Heidelberg, New York: Springer-Verlag), also Lecture Notes in Physics 1991, 385, 230

Tanaka, Y., Nandra, K., Fabian, A. C., et al. 1995, Nature, 375, 659

Turner, T. J., Mushotzky, R. F; Yaqoob, T., et al. 2002, ApJ, 574, L123

Verner, D. A., Yakovlev, D. G., Band, I. M., \& Trzhaskovskaya, M. B. 1993, Atomic Data and Nuclear Data Tables, 55, 233

Yaqoob, T., George, I. M., \& Turner, T. J. 2001, Chandra Grating Observations of Seyfert 1 Galaxies [astro-ph/0111428]

Zdziarski, A. A., Ghisellini, G., George, I. M., et al. 1990, ApJ, 363, L1

Zycki, P. T., \& Czerny, B. 1994, MNRAS, 266, 653

Wilms, J., Reynolds, C. S., Begelman, M. C., et al. 2001, MNRAS, 328, L27 